\title{
A re-evaluation of the enigmatic dinosauriform Caseosaurus crosbyensis from the Late Triassic of Texas, USA and its implications for early dinosaur evolution
}

\author{
Matthew G. Baron and Megan E. Williams \\ Acta Palaeontologica Polonica 63 (1), 2018: 129-145 doi:https://doi.org/10.4202/app.00372.2017
}

The holotype specimen of the Late Triassic dinosauriform Caseosaurus crosbyensis is redescribed and evaluated phylogenetically for the first time, providing new anatomical information and data on the earliest dinosaurs and their evolution within the dinosauromorph lineage. Historically, Caseosaurus crosbyensis has been considered to represent an early saurischian dinosaur, and often a herrerasaur. More recent work on Triassic dinosaurs has cast doubt over its supposed dinosaurian affinities and uncertainty about particular features in the holotype and only known specimen has led to the species being regarded as a dinosauriform of indeterminate position. Here, we present a new diagnosis for Caseosaurus crosbyensis and refer additional material to the taxon-a partial right ilium from Snyder Quarry. Our comparisons and phylogenetic analyses suggest that Caseosaurus crosbyensis belongs in a clade with herrerasaurs and that this clade is the sister taxon of Dinosauria, rather than positioned within it. This result, along with other recent analyses of early dinosaurs, pulls apart what remains of the "traditional" group of dinosaurs collectively termed saurischians into a polyphyletic assemblage and implies that Dinosauria should be regarded as composed exclusively of Ornithoscelida (Ornithischia + Theropoda) and Sauropodomorpha. In addition, our analysis recovers the enigmatic European taxon Saltopus elginensis among herrerasaurs for the first time. This result suggests a greater body-size range for herrerasaurs than previously thought and provides further evidence for their presence in Europe during the Late Triassic. If this hypothesis is correct then this clade of herrerasaurs also represents the first clade of non-dinosaurian dinosauromorphs known to contain large-bodied carnivorous species. The results of our analyses also highlight the distinction between the clades Herrerasauridae and Herrerasauria, as they are currently defined, and necessitate a provisional revival of the latter until future works can better resolve the relationships among these important early taxa.

Key words: Archosauria, Dinosauromorpha, Herrerasauria, phylogeny, Dockum Group, Triassic, Texas, USA. 
Sciences, Natural History Museum, London, Cromwell Road, London SW7 5BD,

UK. Megan E. Williams [mew41@ cam.ac.uk], Department of Earth Science, University of Cambridge, Downing Street, Cambridge, CB2 3EQ, UK.

This is an open-access article distributed under the terms of the Creative Commons Attribution License (for details please see creativecommons.org), which permits unrestricted use, distribution, and reproduction in any medium, provided the original author and source are credited.

PoF Full text $(547.7 \mathrm{kB})$ ।

FoF Supplementary file $(688.5 \mathrm{kB})$ 